\title{
A case report of a deep surgical site infection with Terrisporobacter glycolicus/T. Mayombei and review of the literature
}

\author{
Matthew P. Cheng ${ }^{1,3^{*}}$, Marc-Christian Domingo ${ }^{2}$, Simon Lévesque ${ }^{2}$ and Cedric P. Yansouni ${ }^{1,3}$
}

\begin{abstract}
Background: There are increasing data regarding Terrisporobacter glycolicus as an emerging anaerobic pathogen. However, the few published cases to date usually report it as part of a polymicrobial infection. Here, we describe the first reported monomicrobial surgical site infection with this bacterium. Identification methods, taxonomy, and clinical management of this rarely identified pathogen are also discussed.

Case presentation: A previously healthy 66-year-old sustained an open olecranon fracture of his left arm after trauma. He subsequently underwent open reduction and internal fixation (ORIF), with insertion of an olecranon locking plate and two locking screws. Ten days after surgery, the patient developed increasing pain at the surgical site and noted green discharge from the wound. Culture of the wound discharge yielded grew a pure Grampositive anaerobe identified by the RapidANA ${ }^{\circledR}$ microbial identification system as C. difficile (profile 000010, 99.1\% probability). Reference laboratory testing identified the isolate as T. glycolicus/mayombei (previously designated as Clostridium glycolicum/mayombei) by $16 \mathrm{~S}$ rRNA gene sequencing and as Clostridium glycolicum by MALDI-TOF mass spectrometry. The patient received an 8-week course of moxifloxacin and metronidazole with an excellent clinical response at 12 months' follow-up.
\end{abstract}

Conclusions: We describe the case of a deep surgical site infection with T. glycolicus/mayombei (formerly known as Clostridium glycolicum and Clostridium mayombei, respectively), which extends our knowledge of the clinical spectrum of this pathogen. The isolate was misidentified by phenotypic identification methods.

Keywords: Surgical site infection, Clostridium glycolicum, Terrisporobacter glycolicus, Terrisporobacter mayombei, MALDI-TOF, PCR

\section{Background}

Clostridium spp. are a heterogeneous group of anaerobic, spore-forming, gram-positive rod-shaped bacteria. Over 200 distinct species have been identified, most of which are environmental organisms; few species have been recognized to cause clinical disease [1]. There are increasing data regarding Terrisporobacter glycolicus \& Terrisporobacter mayombei as emerging anaerobic pathogens, yet relatively little is known about their pathogenic potential. Previous

\footnotetext{
* Correspondence: matthew.cheng@mail.mcgill.ca

'Division of Infectious Diseases and Department of Medical Microbiology, Glen site, McGill University Health Centre, 1001 Boulevard Décarie, Room E05. 1811.2, Montreal, Quebec H4A 3J1, Canada

${ }^{3}$ J.D. MacLean Centre for Tropical Diseases, McGill University Health Centre, Montreal, Canada

Full list of author information is available at the end of the article
}

case reports of C. glycolicum (the former designation of $T$. glycolicus) identified the bacteria in cultures of wounds and blood, often in conjunction with other pathogens [2-4]. Although it has been implicated in bloodstream infections, brain abscesses as well as bone and joint infections, its ideal method of identification has yet to be determined.

We describe the first reported monomicrobial surgical site infection with prosthetic material from $T$. glycolicus/T. mayombei. Phenotypic identification systems used in our laboratory at the time of the case identified it as $C$. difficile, but $16 \mathrm{~S}$ ribosomal sequence analysis yielded $T$. glycolicus/T. mayombei with a high degree of certainty. In addition, MALDITOF MS system demonstrated the ability to correctly identify C. glycolicum, under its former designation. 


\section{Case presentation}

A previously healthy 66-year-old man fell off of his bicycle onto dry pavement, and sustained an open olecranon fracture of his left arm. He subsequently underwent open reduction and internal fixation (ORIF), with insertion of an olecranon locking plate and two locking screws. Chlorhexidine-based sterilizing solution and peri-operative cefazolin were used in accordance with our institution's protocols. The procedure was uncomplicated and the patient was discharged home on the third post-operative day.

Ten days after surgery, the patient developed increasing pain at the surgical site and noted green discharge from the wound. He was re-admitted to hospital for surgical drainage of the affected elbow, and piperacillin-tazobactam was prescribed empirically. Culture of the wound discharge yielded anaerobic spore-forming Gram-positive rods in broth media and anaerobic $5 \%$ sheep blood agar, identified by the RapidANA ${ }^{\circ}$ microbial identification system as $C$. difficile (profile 000010, $99.1 \%$ probability). The aerobic cultures were negative. Given the rarity of $C$. difficile joint infections [5, 6], was reported as "Clostridium species" and the isolate was sent to the provincial reference laboratory for speciation. Our patient did not have any evidence of $C$. difficile-associated diarrhea and his stool specimen was negative for $C$. difficile toxin by PCR. He was subsequently treated with intravenous vancomycin and oral metronidazole, pending results of antimicrobial susceptibility testing.

At the Laboratoire de santé publique du Québec (LSPQ), latex agglutination (Microgen Bioproducts Ltd) yielded a positive result indicating a possible $C$. difficile strain. However, according to the manufacturer package insert, C. glycolicum is known to cross react with this reagent. The isolate was identified as C. glycolicum by MALDI-TOF mass spectrometry using the bioMérieux VITEK MS system (IVD database version 2.0). The clinical isolate, designated LSPQ-04251, was definitively identified by $16 \mathrm{~S}$ rRNA gene sequencing using a BigDye method with ABI3130 XL sequencing (Applied Biosystems, Foster City, CA). Pairwise nucleotide sequence similarities of the 16S rRNA gene indicated that the closest known relatives of the isolate were Clostridium glycolicum (99.3\%) (recently reclassified as Terrisporobacter glycolicus), and Clostridium mayombei (99.2 \%) (recently reclassified as Terrisporobacter mayombei) [7]. Based on the 16S rRNA gene sequence comparison, $T$. glycolicus and T. mayombei were genetically highly related, displaying $99.4 \%$ sequence similarities with $0.6 \%$ divergence. No further characterization of isolate LSP-04251 to separate both species T. glycolicus and T. mayombei, (requiring additional analysis of the genomic DNA $G+C$ content, predominant cellular fatty acids and products from peptone yeast extract) was performed [7].
As there is little experience with clinical infections from these bacteria [8], we relied on minimal inhibitory concentration (MIC) determination by Etest ${ }^{\circ}$, according to the manufacturer's application guide, to guide antimicrobial treatment (see Table 1). Treatment was changed to oral moxifloxacin and metronidazole due to logistical barriers to intravenous therapy in this patient and their excellent bioavailability and bone penetration $[9,10]$, for a total of 8 weeks. One year after stopping antibiotics, the patient was well, without signs of recrudescent infection.

\section{Discussion}

Surgical site infections are an important complication of open fractures, and this risk is modulated by several variables including the size of the wound, the complexity of the fracture, and the presence of necrotic soft tissue [11]. Our patient's wound was classified as Gustilo Type II (i.e. open fracture with a laceration $<1 \mathrm{~cm}$ in length without extensive soft tissue damage, flaps, or avulsions) without severe contamination. No antimicrobial prophylaxis was given beyond the surgical procedure. It is most plausible that our patient's wound was infected with $T$. glycolicum/T. mayombei at the time of his initial injury on the pavement. Although we cannot exclude haematogenous seeding from the gastrointestinal tract, this would seem unlikely given the circumstances of the accident, the organism identified and the absence of GI symptoms or underlying disease.

Although not currently assigned formally to the family Peptostreptococcaceae, phylogenetic analyses indicated that the species Terrisporobacter glycolicus and T. mayombei are members of the family Peptostreptococcaceae [7]. To support this classification in the family of Peptostreptococcaceae, Yutin and Galpieri compared a phylogenetic tree for a concatenated set of 50 widespread ribosomal proteins with the trees for beta subunits of the RNA polymerase (RpoB) and DNA gyrase (GyrB) genes and with the $16 \mathrm{~S}$ rRNA-based phylogeny tree. The result of this

Table 1 Minimal inhibitory concentration (MIC; $\mu \mathrm{g} / \mathrm{mL}$ ) of the clinical isolate of T. glycolicus/T. mayombei, as determined by Etest ${ }^{\circledR}$

\begin{tabular}{ll}
\hline Antimicrobial & $\mathrm{MIC}(\mu \mathrm{g} / \mathrm{mL})$ \\
\hline Piperacillin/Tazobactam & 1.5 \\
Penicillin & 1 \\
Metronidazole & 0.5 \\
Meropenem & 1 \\
Clindamycin & 0.125 \\
Cefoxitin & 32 \\
Ceftriaxone & 32 \\
Imipenem & 32 \\
Moxifloxacin & 1 \\
\hline
\end{tabular}


analysis support placement of Clostridium difficile and its close relatives (including species of the genus Terrisporobacter) within the family Peptostreptococcaceae. Hence, some have proposed to rename C. difficile as Peptoclostridium difficile, and to transfer C. glycolicum and $C$. mayombei to this genus as well [1]. This taxonomic controversy remains unresolved for the moment. Thus, based on available phylogenetic data, misidentification of the isolate LSPQ-04251 as C. difficile by phenotypic methods is not unexpected.

Relatively little is known about the pathogenic potential of T. glycolicus/T. mayombei. Elsayed and Zhang first reported a first case of human infection with C. glycolicum in 2007, when a 43 year old woman developed septic shock after a recent bone marrow transplant for relapsed Hodgkin's disease [2]. Their patient was found to have a polymicrobial bactermia with C. glycolicum and Enterococcus spp. She received numerous antibiotics initially and subsequently completed a two-week course of ampicillin, gentamicin and metronidazole with a good clinical response. In 2009, Van Leer et al. described the case of a 62 year-old gentleman who developed a brain abscess with gas formation after otitis media, for which he treated himself with clay in his ear [4]. C. glycolicum was the only organism identified in the patient's blood, but numerous other organisms were recovered from the pus and necrotic tissue from the mastoid. Their patient eventually recovered with residual bilateral hearing loss after surgical debridement of the infection. In 2012, Jiang et al. reported two cases of human infection of C. glycolicum after suffering accidental injuries. Both cases resulted in polymicrobial infections, with one patient developing a coagulasenegative staphylococcal bacteremia requiring six weeks of intravenous antibiotic therapy with vancomycin. Subsequently, Cai et al. described a case of a 67 year-old gentleman with numerous medical co-morbidities who developed acalculous cholecystitis and was found to have a bloodstream infection with C. glycolicum. No other pathogens were identified and the patient responded well to treatment with pipacillin-tazobactam.

\section{Conclusion}

We describe the first reported monomicrobial surgical site infection from T. glycolicus/T. mayombei. Sequence analysis of the 16S rRNA gene offers greater discrimination between genera and, in many cases, species compared to the other bacterial identification methods described in this report. However, MALDI-TOF mass spectrometry also correctly identified C. glycolicum under its former designation and may be a reliable identification method for these pathogens. Our case demonstrates the limitations of phenotypic identification systems for clostridial species, and extends our knowledge of the clinical spectrum of $T$. glycolicus/T. mayombei. Finally, species identification for deep Grampositive anaerobic rod infections is pertinent because antimicrobial susceptibility patterns may be poorly characterised or variable, and mandate susceptibility testing for rarely encountered species.

\section{Key points}

- Terrisporobacter glycolicus/Terrisporobacter mayombi is a rare cause of clinical infection, but has been implicated in brain abscesses, bone and joint infections, as well as bloodstream infections.

- T. glycolicus/mayombi was misidentified as C. difficile by phenotypic and latex agglutination testing. Accurate identification was achieved with both $16 \mathrm{~S}$ rRNA gene sequence analysis and MALDI-TOF MS.

- Identification to the species level for deep Grampositive anaerobic rod infections is potentially clinically pertinent, and rarely encountered species should prompt antimicrobial susceptibility testing.

\section{Abbreviations}

MALDI-TOF: Matrix-assisted laser desorption/ionization - time of flight; MIC: miminal inhibitory concentration; ORIF: Open reduction and internal fixation

Funding

CPY holds a Chercheur-boursier clinicien career award from the Fonds de recherche du Québec - Santé (FRQS).

\section{Availability of data and materials}

Not applicable.

\section{Authors' contributions}

MPC and CPY provided clinical care for the patient. MPC performed literature searches and drafted the article. MCD performed $16 \mathrm{~S}$ ribosomal sequence analysis. SL performed latex agglutination and MALDI-TOF MS analysis. CPY was responsible for the overall content. All authors read and approved the final manuscript.

\section{Competing interests}

SL has a research agreement with bioMérieux Canada for the utilization of VITEK MS system. All other authors declare that they have no competing interests.

Consent for publication

the patient has provided written consent to publish this case report.

Ethics approval and consent to participate

Not applicable.

\section{Author details}

${ }^{1}$ Division of Infectious Diseases and Department of Medical Microbiology, Glen site, McGill University Health Centre, 1001 Boulevard Décarie, Room E05. 1811.2, Montreal, Quebec H4A 3J1, Canada. 'Laboratoire de santé publique du Québec, McGill University Health Centre, Montreal, Canada. ${ }^{3} J . D$. MacLean Centre for Tropical Diseases, McGill University Health Centre, Montreal, Canada.

Received: 25 June 2016 Accepted: 22 September 2016 Published online: 29 September 2016 


\section{References}

1. Yutin N, Galperin MY. A genomic update on clostridial phylogeny: gramnegative spore formers and other misplaced clostridia. Environ Microbiol. 2013;15(10):2631-41.

2. Elsayed S, Zhang K. Clostridium glycolicum bacteremia in a bone marrow transplant patient. J Clin Microbiol. 2007:45(5):1652-4.

3. Jiang W, Abrar S, Romagnoli M, Carroll KC. Clostridium glycolicum wound infections: case reports and review of the literature. J Clin Microbiol. 2009; 47(5):1599-601.

4. Van Leer C, Wensing AM, van Leeuwen JP, Zandbergen EG, Swanink CM. Clostridium glycolicum isolated from a patient with otogenic brain abscesses. Clin Microbiol. 2009;47(2):511-3.

5. Brassinne $\mathrm{L}$, Rodriguez-Villalobos $\mathrm{H}$, Jonckheere $\mathrm{S}$, Dubuc JE, Yombi JC. Early infection of hip joint prosthesis by Clostridium difficile in an HIV-1 infected patient. Anaerobe. 2014:27:96-9.

6. Gregg KS, Alexander KA. Native joint septic arthritis caused by Clostridium difficile in an 11-year old with hemoglobin SS disease. Pediatr Infect Dis J. 2009;28(9):853.

7. Gerritsen J, Fuentes S, Grievink W, van Niftrik L, Tindall BJ, Timmerman HM, et al. Characterization of Romboutsia ilealis gen. nov., sp. nov., isolated from the gastro-intestinal tract of a rat, and proposal for the reclassification of five closely related members of the genus Clostridium into the genera Romboutsia gen. nov., Intestinibacter gen. nov., Terrisporobacter gen. nov. and Asaccharospora gen. nov. Int J Syst Evol Microbiol. 2014;64(Pt 5):1600-16.

8. Cai D, Sorokin V, Lutwick L, Liu W, Dalal S, Sandhu K, et al. C. glycolicum as the sole cause of bacteremia in a patient with acute cholecystitis. Ann Clin Lab Sci. 2012;42(2):162-4.

9. Summersgill JT, Schupp LG, Raff MJ. Comparative penetration of metronidazole, clindamycin, chloramphenicol, cefoxitin, ticarcillin, and moxalactam into bone. Antimicrob Agents Chemother. 1982;21(4):601-3.

10. Malincarne L, Ghebregzabher M, Moretti MV, Egidi AM, Canovari B, Tavolieri $\mathrm{G}$, et al. Penetration of moxifloxacin into bone in patients undergoing total knee arthroplasty. J Antimicrob Chemother. 2006:57(5):950-4.

11. Gustilo RB, Mendoza RM, Williams DN. Problems in the management of type III (severe) open fractures: a new classification of type III open fractures. J Trauma. 1984;24(8):742-6

\section{Submit your next manuscript to BioMed Central and we will help you at every step:}

- We accept pre-submission inquiries

- Our selector tool helps you to find the most relevant journal

- We provide round the clock customer support

- Convenient online submission

- Thorough peer review

- Inclusion in PubMed and all major indexing services

- Maximum visibility for your research

Submit your manuscript at www.biomedcentral.com/submit 